Int. J. Odontostomat., 9(3):385-391, 2015.

\title{
Análisis de Patrones de Cierre Velofaríngeo en Pacientes con Labio y Paladar Hendido
}

\author{
Pattern Analysis of Velopharyngeal Closure in Patients With Cleft Lip and Palate
}

\begin{abstract}
María Priscila Hernández Rocha*; Benjamín Sánchez Trocino*; Javier de la Fuente Hernández*; María del Carmen Villanueva Vilchis" ${ }^{* *}$ Jacinto Armando Díaz Acevedo*; Gabriela Vilar Pineda** \& Fernando Tenorio Rocha
\end{abstract}

HERNÁNDEZ, R. M. P.; SÁNCHEZ, T. B.; DE LA FUENTE, H. J.; VILLANUEVA, V. M. C.; DÍAZ, A. J. A.; VILAR, P. G. \& TENORIO, R. F. Análisis de patrones de cierre velofaríngeo en pacientes con labio y paladar hendido. Int. J. Odontostomat., 9(3):385-391, 2015.

RESUMEN: El objetivo fue identificar los patrones de Cierre Velofaríngeo (CVF) en pacientes con Paladar Hendido (PH) y Labio y Paladar Hendido (LPH) que acuden a la Clínica de Atención Integral de Pacientes con Labio y Paladar Hendido (CAIPLPH) de la Escuela Nacional de Estudios Superiores, Unidad León (ENES, León). Estudio transversal, con una muestra por conveniencia de 79 pacientes entre 4 y 10 años de edad. Las variables incluidas fueron el patrón de CVF, tipo de hendidura, edad del paciente y el antecedente de intervención quirúrgica previa para el cierre de la hendidura palatina. A los pacientes se les realizó valoración clínica y nasofibroscopía por examinadores previamente estandarizados para conocer si existe una relación entre edad y patrón de CVF, entre el tipo de hendidura y patrón de CVF y una relación entre el patrón de CVF y el antecedente quirúrgico, utilizando una prueba bivariada de Chi cuadrada. Se examinaron 46 hombres y 33 mujeres con una media de edad 6,6 años. La frecuencia de los patrones de CVF fueron coronal 8,86\%, sagital 67,08\%, circular 13,92\% y circular con rodete de Passavant 10,12\%. Se observó una diferencia estadística significativa entre el patrón de CVF y el tipo de hendidura $\left(X^{2}=53,93, p<0,001\right)$. Las diferentes formas en que se pueden presentar las hendiduras de paladar $y$ labiopalatinas influyen en la función del CVF ya que se puede presentar un patrón distinto en cada caso. El patrón de CVF no se ve afectado por la edad ni por el antecedente quirúrgico de cierre de la hendidura palatina.

PALABRAS CLAVE: insuficiencia velofaríngea, paladar hendido, labio y paladar hendido.

\section{INTRODUCCIÓN}

La Insuficiencia Velofaríngea (IVF) es un trastorno funcional, el cual ocurre cuando no existe un cierre entre las paredes faríngeas laterales, posteriores y el velo del paladar durante la fonación y la deglución, donde no existe una separación perfecta entre la cavidad nasal y la cavidad oral por lo cual se merma la calidad de estas funciones (Naiman \& Disant, 2006). Este trastorno funcional además puede causar habla hipernasal, emisión y turbulencia nasal y regurgitación nasal de líquidos (Lu et al., 2012).

El cierre del Esfínter Velofaríngeo (EVF) es esencial para la producción del habla. Su apropiado funcionamiento depende de los músculos del paladar y de la faringe. Es un mecanismo esfinteriano con dos componentes: velar y faríngeo. El movimiento velar está determinado por la acción del músculo elevador del velo. El movimiento faríngeo depende de la contracción del músculo constrictor superior de la faringe y del músculo palatofaríngeo. Cuando uno de los dos componentes es deficiente, se produce un defecto de cierre que altera cuatro funciones principales: fonación, deglución, respiración y audición (Prada Madrid et al., 2010).

La fisiología del EVF implica el retroceso y el ascenso del velo del paladar por la acción del músculo elevador del velo del paladar, la principal masa muscular del paladar blando. La contracción de los músculos palatogloso y palatofaríngeo en sentido medial, que sirve probablemente para traccionar en sentido inferior del velo del paladar, se opone a la acción ascendente del elevador del velo del paladar generando

\footnotetext{
* Alumna de la Licenciatura en Odontología, Escuela Nacional de Estudios Superiores, Universidad Nacional Autónoma de México, León, México.

"* Profesor Investigador de la Escuela Nacional de Estudios Superiores, Universidad Nacional Autónoma de México, León, México.
} 
una constricción que en la deglución y fonación cierra el EVF (Conessa et al., 2005).

Las causas de la IVF son diversas, y van desde la parálisis (secundaria a traumatismos o a una intervención quirúrgica que afecte al velo, la rinofaringe o la base del cráneo) hasta las anomalías anatómicas congénitas del velo (Gart \& Gosain, 2014).

Los pacientes con $\mathrm{PH}$ o con LPH desarrollan cierto grado de IVF debido a la disposición anormal de las estructuras musculares adyacentes a la hendidura. El PH y el LPH reparado o no reparado representan las causas más comunes de IVF. En el grupo de pacientes con antecedente quirúrgico de cierre de la hendidura, la IVF se debe a un alargamiento insuficiente del velo del paladar en el momento de la palatoplastia primaria, la función anormal del músculo elevador, la musculatura alterada y la contractura cicatrizal del velo del paladar (Gart \& Gosain).

Los pacientes con $\mathrm{PH}$ o LPH se someten a una cirugía palatina cuyo principal objetivo es el cierre del paladar para crear un paladar anatómica y funcionalmente óptimo para así mejorar la deglución, la fonación y el correcto crecimiento y desarrollo del maxilar. Con el fin de producir el habla normal, el paciente debe tener competencia velofaríngea, definida como la capacidad para cerrar completamente el EVF que separa la región orofaríngea de la región nasofaríngea. Por su parte, la IVF se observa comúnmente en pacientes con $\mathrm{PH}$ o LPH después del cierre primario de la hendidura palatina.

Patrones de cierre del velofaríngeo. Es la conducta dinámica de los músculos involucrados en el cierre del EVF. Se han descrito varios grupos en función de los movimientos de las cuatro paredes durante el cierre (dos laterales, una anterior y una posterior). Skolnick et al. (1973) y Croft et al. (1981) describieron inicialmente diferentes patrones de cierre velofaríngeo. De acuerdo al movimiento de las estructuras durante la fonación es posible considerar la existencia de cuatro patrones de CVF: coronal, sagital, circular y circular con rodete de Passavant (Naiman \& Disant).

- Coronal: es el modo más frecuente $(55 \%$ de las personas). Se produce cuando el cierre del esfínter se debe sobre todo al movimiento posterior del paladar blando. En este grupo, una gran parte de la pared faríngea contacta con el paladar blando y en ese punto se asocia un pequeño movimiento de la pared faríngea lateral en sentido medial.
- Sagital: el cierre sagital se identifica cuando la mayoría del movimiento de cierre se realiza por las paredes laterales; la parte posterior del velo no supone más que un componente accesorio para el cierre. Este tipo se encuentra en el $10-15 \%$ de las personas.

- Circular: en este tipo de cierre las paredes faríngeas y el velo del paladar participan en conjunto y de forma equivalente al cierre del esfínter. Esto crea un círculo que se cierra de forma progresiva. Este grupo representa el $10-20 \%$ de la población.

- Circular con rodete de Passavant: en esta situación, las cuatro paredes faríngeas intervienen en el cierre del EVF, pero particularmente se observa una prominencia en la superficie anterior de la faringe (pared posterior del EVF) que corresponde a una hipertrofia fisiológica denominada Rodete de Passavant para permitir el cierre. Este grupo representa el $15-20 \%$ de la población. El rodete de Passavant se describió en 1868 por este autor como un movimiento de los tejidos faríngeos durante el habla (Conessa et al.).

La evaluación de un paciente que presente evidencias de insuficiencia velofaríngea se debe comenzar con una historia clínica y una exploración física. En particular, la aparición de anomalías del habla, historia previa de labio y/o paladar hendido así como también cualquier cirugía previa palatofaringea deben ser tomadas en cuenta (Gart \& Gosain).

El examen físico, comienza con la inspección desde los labios, en busca de cualquier cicatriz secundaria a una intervención previa y se continúa con el velo del paladar y la faringe. En el velo del paladar se buscará alguna dehiscencia medial, se observará la simetría del velo y de los pilares anteriores y posteriores; En la faringe se observará su volumen. El examen físico también incluye valorar la fonación, en la cual se evalúa la contracción de la pared faríngea y los movimientos ascendentes, laterales y transversales de todos los elementos que conforman el EVF (Gart \& Gosain).

Existen varios auxiliares de diagnóstico que nos permiten valorar el cierre del EVF los cuales se pueden clasificar en directos o indirectos y en estáticos o dinámicos. En los protocolos de diagnóstico se han llegado a usar recursos imagenológicos como la Tomografía Computadorizada (TC) y la Resonancia Nuclear Magnética (RNM), con el inconveniente de que las imágenes obtenidas son estáticas y no permiten la valoración dinámica y funcional del EVF. La 
nasofibroscopía junto con la videofluoroscopia, están consideradas como las técnicas mas adecuadas para la valoración directa y dinámica de los movimientos del EVF (Gart \& Gosain).

La nasofibroscopía consiste en introducir una fibra óptica flexible a través de una fosa nasal, hasta llegar a la nasofaringe, lo que permite la visualización directa del EVF, de sus estructuras y de los movimientos realizados durante el habla y la deglución, desde una vista superior (Prada Madrid et al.). La función del EVF se estudia en reposo y después en movimientos durante la fonación al hacer que el paciente pronuncie frases normalizadas que incluyen fonemas orales $y$ nasales (Gart \& Gosain).

En la CAIPLPH de la ENES, León, se atienden a 209 pacientes que padecen esta anomalía congénita. La clínica cuenta con una moderna infraestructura y equipamiento que permite ofrecer a los pacientes una atención especializada de primer nivel para brindarles tratamientos específicos de acuerdo a sus necesidades clínicas. El diagnóstico de la IVF y de los patrones de CVF en este tipo de pacientes es útil para determinar el grado de IVF y las necesidades quirúrgicas que tienen que ser atendidas para la rehabilitación del paciente.

El objetivo fue conocer los patrones de CVF en los pacientes con $\mathrm{PH}$ y LPH de edades entre 4 y 10 años que reciben atención médica especializada en la CAIPLPH de la ENES León. Los objetivos específicos fueron determinar si existe una relación entre la edad y el patrón de CVF, entre el tipo de hendidura y el patrón de CVF y una relación entre el patrón de CVF y el antecedente de una cirugía previa de cierre de la hendidura palatina.

\section{MATERIAL Y MÉTODO}

Fue un estudio transversal, con un universo de estudio constituido por 79 pacientes por conveniencia que se sometieron al protocolo de diagnóstico de IVF de la ENES León, el cual consiste en una nasofibroscopía directa, la cual se realiza con un Rinolaringoscopio de video portátil marca Hawk (Hangzhou Hawk Optical Electronic Instruments, Co. Ltd). Los criterios de inclusión fueron pacientes inscritos a la CAIPLPH, pacientes con PH o con LPH. Se excluyeron aquellos pacientes que no cumplieron el criterio de edad. Las variables que se utilizaron fueron el tipo de hendidura evaluada clínicamente con base a la clasificación anatómica, la edad del paciente registrada en años y el patrón de CFV observado a través de nasofribroscopías realizadas por tres diferentes especialistas previamente estandarizados que participan en la CAIPLPH obteniendo imágenes en archivos digitales para su posterior análisis. El análisis de los resultados fue llevado a cabo por dos especialistas y un alumno de la clínica de CAIPLPH previamente estandarizados. La información fue registrada en una hoja de Excel 2010 (Microsoft Office Professional. U.S). El análisis estadístico se realizó con ayuda del software SPSS 2013 versión 22 (IBM Corporation. U.S) para conocer si existe una relación entre la edad y el patrón de CVF, entre el tipo de hendidura y el patrón de CVF y una relación entre el patrón de CVF y el antecedente quirúrgico, utilizando una prueba bivariada de Chi cuadrada, con un valor de significancia de 0,05.

Debido a que todos los pacientes eran menores de edad, se les pidió a sus padres o tutores, firmar un consentimiento informado en donde se autorizó la participación de los pacientes en este estudio.

\section{RESULTADOS}

La muestra se conformó de 79 pacientes, de los cuales 24 sólo presentaban $\mathrm{PH}$ y 55 presentaban LPH. La media de edad observada fue de $6,6 \pm 1,92$ años.

De acuerdo al tipo de hendidura, la distribución observada fue como la siguiente: 24 pacientes con $\mathrm{PH}$, los cuales se subdividieron en: 18 pacientes con Paladar Hendido Secundario (PHS), 2 con Paladar Hendido Submucoso (PHSM), uno con Paladar Hendido Completo Unilateral Derecho (PHCUD), dos pacientes con Paladar Hendido Completo Unilateral Izquierdo (PHCUI) y un paciente con Paladar Hendido Completo Bilateral (PHCB). Asimismo, los 55 pacientes con LPH se subdividieron en 24 pacientes con Labio y Paladar Hendido Completo Bilateral (LPHCB), 13 con Labio y Paladar Hendido Completo Unilateral Derecho (LPHCUD), 17 con Labio y Paladar Hendido Completo Unilateral Izquierdo (LPHCUI) y únicamente un paciente con Labio Hendido Incompleto Bilateral con Paladar Hendido Completo Bilateral (LHIB-PHCB). De los 24 pacientes que presentaban sólo $\mathrm{PH}$, diez no habían sido sometidos a tratamiento quirúrgico para cierre de la hendidura palatina, mientras que en los pacientes que presentaban LPH, solamente 5 pacientes 
HERNÁNDEZ, R. M. P.; SÁNCHEZ, T. B.; DE LA FUENTE, H. J.; VILLANUEVA, V. M. C.; DíAZ, A. J. A.; VILAR, P. G. \& TENORIO, R. F. Análisis de patrones de cierre velofaríngeo en pacientes con labio y paladar hendido. Int. J. Odontostomat., 9(3):385-391, 2015.

no habían sido sometidos al tratamiento quirúrgico de cierre de la hendidura palatina. No se observó diferencia estadística significativa de acuerdo al tipo de hendidura por edad $\left(X^{2}=48,58 p=0,225\right)$ (Tabla I).

En cuanto a la frecuencia de patrones de cierre velofaríngeo observada, la distribución fue la siguiente: patrón de cierre coronal en 7 pacientes $(8,86 \%)$, patrón de cierre sagital en 53 pacientes $(67,08 \%)$, patrón de cierre circular en 11 pacientes $(13,92 \%)$ y patrón de cierre circular con rodete de Passavant en 8 pacientes $(10,12 \%)$. Se observó diferencia estadística significativa de acuerdo al patrón de CVF $(p<0,001)$ (Tabla II).

De acuerdo al tipo de hendiduras palatinas, la frecuencia de los patrones de cierre que se observaron fueron los siguientes: En el grupo de pacientes con PHS (18 pacientes) ningún paciente presentó un patrón de CVF coronal, 10 pacientes $(55,55 \%)$ presentaron un patrón de CVF sagital, 2 pacientes $(11,11 \%)$ presentaron un patrón de CVF circular y 6 pacientes $(33,33 \%)$ presentaron un patrón de CVF circular con rodete de Passavant. En el grupo de pacientes con PHSM (2 pacientes) únicamente se presentó el patrón de CVF sagital (100\%). En el grupo de pacientes con PHCUD (1 paciente) sólo se presentó el patrón de CVF circular con rodete de Passavant (100\%). En el grupo de pacientes con PHCUI (2 pacientes) se presentó el patrón de CVF sagital en un paciente $(50 \%)$ y el patrón de CVF circular (50\%). En el grupo de PHCB (1 paciente) se observó el patrón de CVF circular (100\%). Se observó diferencia estadística significativa de acuerdo al patrón de CVF en relación al tipo de hendidura $(X 2=53,93, p<0,001)$ (Tabla III).

En relación al tipo de hendiduras palatinas con labio, la frecuencia de patrones de CVF que se observaron fueron los siguientes: En el grupo de pacientes con LPHCB (24 pacientes) el 100\% presentó el patrón de CVF sagital. En el grupo de pacientes con LPHCUD (13 pacientes) el patrón de CVF coronal se observó en 3 pacientes $(23,07 \%)$. El patrón de CVF sagital se observó en 6 pacientes $(46,15 \%)$. El patrón de CVF circular se observó en 3 pacientes $(23,07 \%)$. El patrón de CVF circular con rodete de Passavant se observó en 1 paciente $(7,69 \%)$. En el grupo de pacientes con LPHCUI (17 pacientes) se observó el patrón de CVF coronal en 4 pacientes (23,52\%). El patrón de CVF sagital se observó en 9 pacientes (52,94\%). El patrón de CVF circular se observó en 4 pacientes $(23,52 \%)$. En el grupo conformado por un solo paciente presentaba LHIB-PHCB el patrón de CVF que se observó fue sagital (100\%). Se observó diferencia estadística significativa de acuerdo al patrón de CVF en relación al tipo de hendidura $\left(X^{2}=53,93 p<0,001\right)$ (Tabla III).

Tabla II. Frecuencia de patrones de cierre velofaríngeo.

\begin{tabular}{lcc}
\hline Patrón & $\mathbf{n}$ & Porcentaje \\
\hline Coronal & 7 & $8,86 \%$ \\
Sagital & 53 & $67,08 \%$ \\
Circular & 11 & $13,92 \%$ \\
Circular C/RP & 8 & $10,12 \%$ \\
\hline$p<0,001$. & &
\end{tabular}

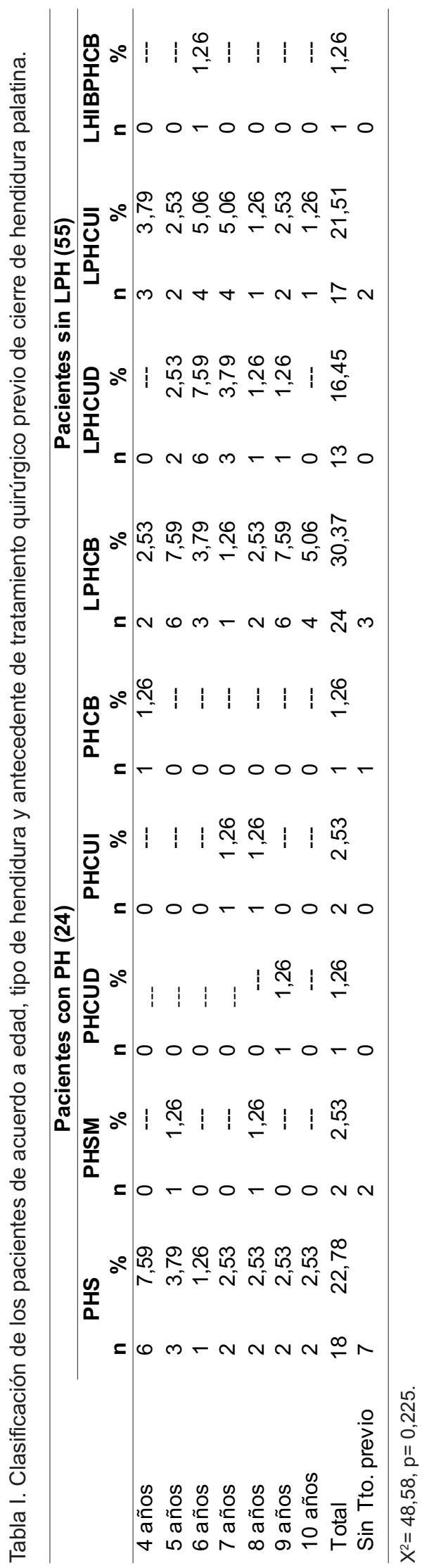




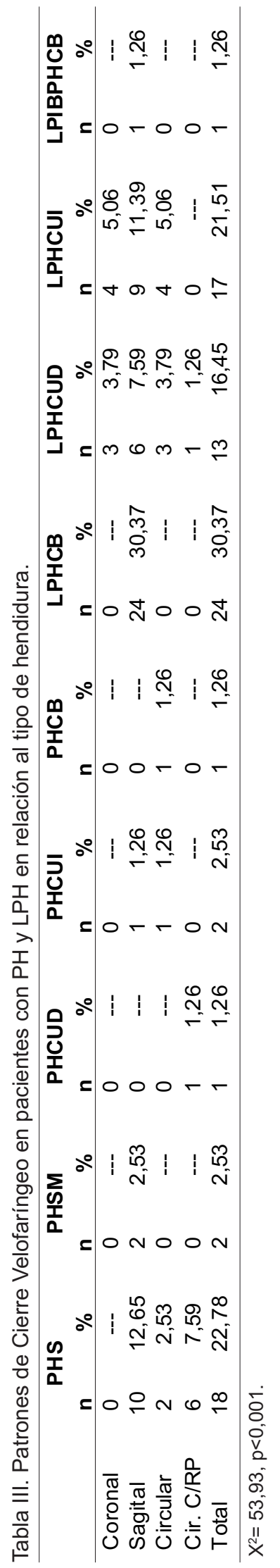

De acuerdo a la edad de los pacientes y tomando en cuenta el tipo de hendidura que presentaron, el comportamiento en la frecuencia de patrones de CVF que se observó fue el siguiente: Pacientes de 4 años (12 pacientes) con hendidura de PH 6 sagitales y un circular, con hendidura de LPH 1 coronal, 3 sagitales y 1 circular. Pacientes de 5 años (14 pacientes) con hendidura de $\mathrm{PH} 3$ sagitales y 1 circular, con hendidura de LPH 1 coronal, 8 sagitales y 1 circular con rodete de Passavant. Pacientes de 6 años (15 pacientes) con hendidura de $\mathrm{PH} 1$ sagital, con hendidura de LPH 4 coronales, 9 sagitales y 1 circular. Pacientes de 7 años (11 pacientes) con hendidura de $\mathrm{PH} 1$ sagital, 1 circular y 1 circular con rodete de Passavant, con hendidura de LPH 5 sagitales y 3 circulares. Pacientes de 8 años (8 pacientes) con hendidura de PH 1 sagital, 1 circular y 2 circular con rodete de Passavant, con hendidura de LPH 4 sagitales. Pacientes de 9 años (12 pacientes) con hendidura de PH 3 circular con rodete de Passavant, con hendidura de LPH 1 coronal, 7 sagitales y 1 circular. Pacientes de 10 años (7 pacientes) con hendidura de PH 1 sagital y 1 circular con rodete de Passavant, con hendidura de LPH 4 sagitales y 1 circular. No se observó diferencia estadística significativa de acuerdo al tipo de CVF por edad $\left(X^{2}=21,26, p=0,266\right)$ (Tabla IV).

Tabla IV. Patrones de Cierre Velofaríngeo en relación al tipo de hendidura y a la edad de los pacientes.

\begin{tabular}{lcccccccc}
\hline & \multicolumn{2}{c}{ Coronal } & \multicolumn{2}{c}{ Sagital } & \multicolumn{2}{c}{ Circular } & \multicolumn{2}{c}{ Cir. C/RP } \\
\cline { 2 - 8 } & PH & LPH & PH & LPH & PH & LPH & PH & LPH \\
\hline 4 años & 0 & 1 & 6 & 3 & 1 & 1 & 0 & 0 \\
5 años & 0 & 1 & 3 & 8 & 1 & 0 & 0 & 1 \\
6 años & 0 & 4 & 1 & 9 & 0 & 1 & 0 & 0 \\
7 años & 0 & 0 & 1 & 5 & 1 & 3 & 1 & 0 \\
8 años & 0 & 0 & 1 & 4 & 1 & 0 & 2 & 0 \\
9 años & 0 & 1 & 0 & 7 & 0 & 1 & 3 & 0 \\
10 años & 0 & 0 & 1 & 4 & 0 & 1 & 1 & 0 \\
Total & 0 & 7 & 13 & 40 & 4 & 7 & 7 & 1 \\
\hline
\end{tabular}

$X^{2}=21,26, p=0,266$.

En cuanto al patrón de CVF observado si los pacientes fueron operados previamente, se observó que ninguno de los pacientes con patrón de CVF coronal fueron operados mientras que el porcentaje que presenta este patrón es de 10,3\% en los pacientes operados, por otra parte, $60 \%$ de los pacientes no operados presentan un patrón de CVF sagital, mientras que estos resultados son equivalentes a $68,8 \%$ en los pacientes operados. En cuanto a la distribución de los pacientes con patrón de CVF circular, $6,7 \%$ se presenta en los pacientes no operados y $15,6 \%$ en los operados; por último, los pacientes con patrón de CVF circular con Rodete de Passavant, se presentan en un $26,7 \%$ de los pacientes no operados y en $6,2 \%$ de los operados. No se observódiferencia estadística significativa entre el patrón de CVF observado con respecto a si el paciente fue operado previamente $\left(X^{2}=5,942, p=0,114\right)($ Tabla V).

Tabla V. Patrones de Cierre Velofaríngeo en relación a si los pacientes fueron operados.

\begin{tabular}{llccccc}
\hline \multirow{2}{*}{ Patrón } & \multicolumn{2}{c}{ No operado } & \multicolumn{2}{c}{ Operado } & \multicolumn{2}{c}{ Total } \\
\cline { 2 - 7 } & $\mathbf{n}$ & $\mathbf{\%}$ & $\mathbf{n}$ & $\mathbf{\%}$ & $\mathbf{n}$ & $\mathbf{\%}$ \\
\hline Coronal & 0 & 0 & 7 & 10,3 & 7 & 8,9 \\
Sagital & 9 & 60,0 & 44 & 68,8 & 53 & 67,1 \\
Circular & 1 & 6,7 & 10 & 15,6 & 11 & 13,9 \\
Cir. C/RP & 4 & 26,7 & 4 & 6,2 & 8 & 10,1 \\
Total & 15 & 100 & 64 & 100 & 79 & 100 \\
\hline
\end{tabular}

$\mathrm{X}^{2}=5,942, p=0,114$. 


\section{DISCUSIÓN}

En este estudio se determinaron los patrones de CVF en una muestra de pacientes con PH y LPH pertenecientes a la CAIPLPH de la ENES, León, observando que de los cuatro patrones de CVF el que se presenta con mayor frecuencia es el cierre sagital. La nasofibroscopía permitió una visualización directa del movimiento velofaríngeo durante el habla. Prada Madrid et al., reportaron que no existe ninguna relación directa entre los patrones de CVF entre pacientes sanos y pacientes con $\mathrm{PH}$ o LPH, en su estudio reportaron que de los cuatro patrones de CVF, sólo el cierre coronal, circular y circular con rodete de Passavant fueron identificados, mencionando además que el cierre sagital no se observó en ninguno de los pacientes de su muestra, y que el cierre circular con rodete de Passavant se observa constantemente en los pacientes con hendidura palatina ya que tienden a desarrollar un mayor movimiento del rodete, tanto en desplazamiento como la intensidad del mismo. En nuestro estudio si fue observada una relación estadística significativa entre el patrón de CVF con respecto al tipo de hendidura, sin embargo, cabe mencionar que únicamente se incluyeron pacientes con la anomalía congénita de $\mathrm{PH}$ y LPH sin incluir pacientes sanos; lo que también se puede relacionar a la presencia de patrón de CVF sagital en el $67,08 \%$ de los casos.

Prada Madrid et al. también relacionaron la presencia o no de insuficiencia velofaríngea de acuerdo al patrón de CVF, sin embargo no encontraron ninguna diferencia. En el estudio que realizamos no se realizó un diagnóstico de IVF debido a que la muestra se realizó en pacientes con $\mathrm{PH}$ y LPH e incluso se incluyó pacientes que ya tenían antecedente de cierre quirúrgico de la hendidura palatina.

Es fundamental resaltar la importancia terapéutica que tiene el poder determinar el tipo de patrón de CVF que se presenta en los casos de pacientes con IVF con el fin de planificar personalmente el tratamiento quirúrgico corrector con base a dichos hallazgos.

Lu et al. (2006) y Chen et al. (2011) mencionaron algunos factores relacionados con la presencia de competencia velofaríngea en pacientes que ya habían sido sometidos a un tratamiento quirúrgico de cierre de la hendidura palatina. Los resultados del presente estudio indican que el antecedente quirúrgico de cierre de hendidura no tiene una influencia en el patrón de CVF. Al respecto, Mølsted (1999) sugirió que aunque los pacientes con LPH y PH fueran tratados con el mismo procedimiento quirúrgico el resultado puede diferir considerablemente, debido a la gran variabilidad de la forma craneofacial y por la afectación individual del PH y LPH en cada paciente, que es una característica que logramos observar en este estudio, ya que se observó una diferencia estadística significativa entre el tipo de hendidura y el patrón de CVF.

\section{CONCLUSIONES}

Las diferentes formas en que se pueden presentar las hendiduras de $\mathrm{PH}$ y LPH influyen en la función del CVF ya que se puede presentar un patrón distinto en cada caso. El patrón de CVF no se ve afectado por la edad y por el antecedente quirúrgico de cierre de la hendidura palatina.

\section{AGRADECIMIENTOS}

Este estudio se realizó gracias al apoyo de un proyecto PAPIME PE205914 de la Dirección General de Asuntos del Personal Académico UNAM.

HERNÁNDEZ, R. M. P.; SÁNCHEZ, T. B.; DE LA FUENTE, H. J.; VILLANUEVA, V. M. C.; DÍAZ, A. J. A.; VILAR, P. G. \& TENORIO, R. F. Pattern analysis of velopharyngeal closure in patients with cleft lip and palate. Int. J. Odontostomat., 9(3):385-391, 2015.

ABSTRACT: The objective of the study was to identify patterns of velopharyngeal closure (CVF) in patients with Cleft Palate $(\mathrm{PH})$ and cleft lip and palate (LPH) attending the Clinic of Comprehensive Attention of Patients with Cleft Lip and Palate (CAIPLPH) of the Escuela Nacional de Estudios Superiores, Unidad León (ENES, León). A cross-sectional study and convenience sample of 79 patients was carried out, including those aged between 4 and 10 years. The variables included were: CVF pattern, kind of cleft, the patient's age and history of previous surgery for closure of the palatal cleft. Patients underwent clinical evaluation and nasofibroscopy by examiners previously standardized to know if there is a relationship between age and CVF pattern, between kind of cleft and pattern of CVF and a relationship between the pattern of CVF and patient's history of previous surgery, using a $X^{2}$ test bivariate square. Forty-six men and 33 women with a mean age 6.6 years were examined. Frequency patterns CVF: $8.86 \%$ coronal, sagittal $67.08 \%$, circular $13.92 \%$ and a circular with Passavant ridge $10.12 \%$. A statistically significant 
difference between the pattern of CVF and the kind of cleft $\left(X^{2}=53.93, p<0.001\right)$ was observed. The different ways in which the $\mathrm{PH}$ and $\mathrm{LPH}$ clefts can present influence the function of CVF as it can present a different pattern in each case. CVF pattern is unaffected by age or by the previous surgical closure.

KEY WORDS: velopharyngeal closure, cleft palate, cleft lip and palate.

\section{REFERENCIAS BIBLIOGRÁFICAS}

Chen, Q.; Zheng, Q.; Shi, B.; Yin, H.; Meng, T. \& Zheng, G. N. Study of relationship between clinical factors and velopharyngeal closure in cleft palate patients. J. Res. Med. Sci., 16(7):945-50, 2011.

Conessa, C.; Hervé, S.; Goasdoué, P.; Martigny, E.; Baudelle, E. \& Poncet, J. L. Insuficiencia velofaríngea. Otorrinolaringol., 20-618-A-10, 2005.

Croft, C. B.; Shprintzen, R. J. \& Rakoff, S. J. Patterns of velopharyngeal valving in normal and cleft palate subjects: a multi-view videofluoroscopic and nasendoscopic study. Laryngoscope, 91(2):265-71, 1981.

Gart, M. S. \& Gosain, A. K. Surgical management of velopharyngeal insufficiency. Clin. Plast. Surg., 41(2):253-70, 2014.

Lu, Y.; Shi, B.; Zheng, Q.; Wang, Z. Y. \& Hu, Q. G. Logistic regression analysis on factors associated with velopharyngeal competence after primary repair of cleft palate. Chin. J. Aesthet. Med., 15(11):1279-81, 2006.

Lu, C.; Arriagada, G. C.; Pruzzo, Ch. E.; Gallo, J. C. \& Bley, P. V. Insuficiencia velofaríngea en cirugía adenoamigdalina. Rev. Otorrinolaringol. Cir. Cabeza Cuello, 72(2):139-44, 2012.

Mølsted, K. Treatment outcome in cleft lip and palate: issues and perspectives. Crit. Rev. Oral Biol. Med., 10(2):22539, 1999.

Naiman, A. N. \& Disant, F. Chirurgie de l'insuffisance vélaire. Techniques chirurgicales - Tête et cou, 1(1):1-15, 2006.

Prada Madrid, J. R.; Garcia Venegas, T.; Echeverri Brando, M. P. \& Tavera Herrera, M. C. Patrones de cierre velofaringeo: Estudio comparativo entre población sana y pacientes con paladar hendido. Cir. Plást. Iberolatinoam., 36(4):305-12, 2010.

Skolnick, M. L.; McCall, G. N. \& Barnes, M. The sphincteric mechanism of velopharyngeal closure. Cleft Palate J., 10:286-305, 1973.

\author{
Dirección para Correspondencia: \\ Benjamín Sánchez Trocino \\ Profesor Investigador \\ Escuela Nacional de Estudios Superiores \\ Universidad Nacional Autónoma de México \\ León \\ MÉXICO
}

Email: drsancheztrocino@enes.unam.mx

Recibido: 20-03-2015

Aceptado: 08-10-2015 